\title{
Foveal hemorrhage in an eye with foveal hypoplasia associated with albinism
}

\author{
This article was published in the following Dove Press journal: \\ Clinical Ophthalmology \\ 5 September 2014 \\ Number of times this article has been viewed
}

\section{Naonori Masuda \\ Taiji Hasegawa \\ Mariko Yamashita \\ Nahoko Ogata}

Department of Ophthalmology, Nara Medical University, Nara, Japan
Correspondence: Nahoko Ogata

Department of Ophthalmology, Nara Medical University, 840 Kashihara City,

Nara, 634-8522, Japan

Tel +81744298884

Fax +81744238032

Email ogata@naramed-u.ac.jp
Abstract: Oculocutaneous albinism is a group of congenital disorders caused by alterations of melanin biosynthesis. We report our findings in a patient with oculocutaneous albinism who presented with foveal hypoplasia and a foveal hemorrhage. A 48-year-old man noted a dark spot in the middle of the visual field of his right eye. He had depigmented skin, white hair, white eyebrows, and white cilia. He also had horizontal nystagmus and depigmented irides. His best-corrected visual acuity was $2 / 100$ with -14.0 diopters in the right eye and $3 / 100$ with -5.0 diopters in the left eye. Ophthalmoscopy showed diffuse depigmentation in both eyes and a foveal hemorrhage in the right eye. Optical coherence tomography showed the absence of a foveal pit in both eyes and a subretinal hyperreflective lesion corresponding to the foveal hemorrhage in the right eye. Fluorescein angiography showed that the retinal and choroidal vessels were relatively hypofluorescent because of the lack of a blocking effect of the pigments in the retinal pigment epithelium. Fluorescein angiography and indocyanine green angiography did not show any evidence of choroidal neovascularization in either eye. The foveal hemorrhage in the right eye spontaneously regressed and finally resolved at 3 months after onset. At the final examination, the patient reported that his vision had recovered. A foveal hemorrhage is a rare condition in an eye with foveal hypoplasia associated with albinism. The hemorrhage may be related to high myopia and also to the hypoplasia of the fovea associated with albinism.

Keywords: albinism, foveal hemorrhage, foveal hypoplasia, simple hemorrhage

\section{Introduction}

Albinism is a group of congenital disorders in which melanin biosynthesis is altered, and it affects approximately one in 4,000 people. $^{1}$ The condition can be broadly divided into two categories, ie, oculocutaneous albinism and ocular albinism. In oculocutaneous albinism, there is a reduction or absence of melanin in the hair, skin, and eyes, whereas in ocular albinism, the melanin deficiency is limited to the eyes. ${ }^{2}$

The ocular manifestations associated with albinism include nystagmus, depigmented irides, foveal hypoplasia, macular transparency, strabismus, and high myopia. ${ }^{3}$ However, there has been only one report of a foveal hemorrhage in eyes with albinism. ${ }^{4}$ We report our findings in a patient with oculocutaneous albinism who had a foveal hemorrhage in an eye with foveal hypoplasia.

\section{Case report}

A 48-year-old man consulted our hospital because he had noted a dark spot in the middle of the visual field of his right eye of 2 weeks' duration. Our examination showed that he had depigmented skin, white hair, white eyebrows, and white cilia (Figure 1A). He had horizontal nystagmus and depigmented irides. His best-corrected visual acuity was $2 / 100$ with -14.0 diopters in the right eye and 3/100 with -5.0 


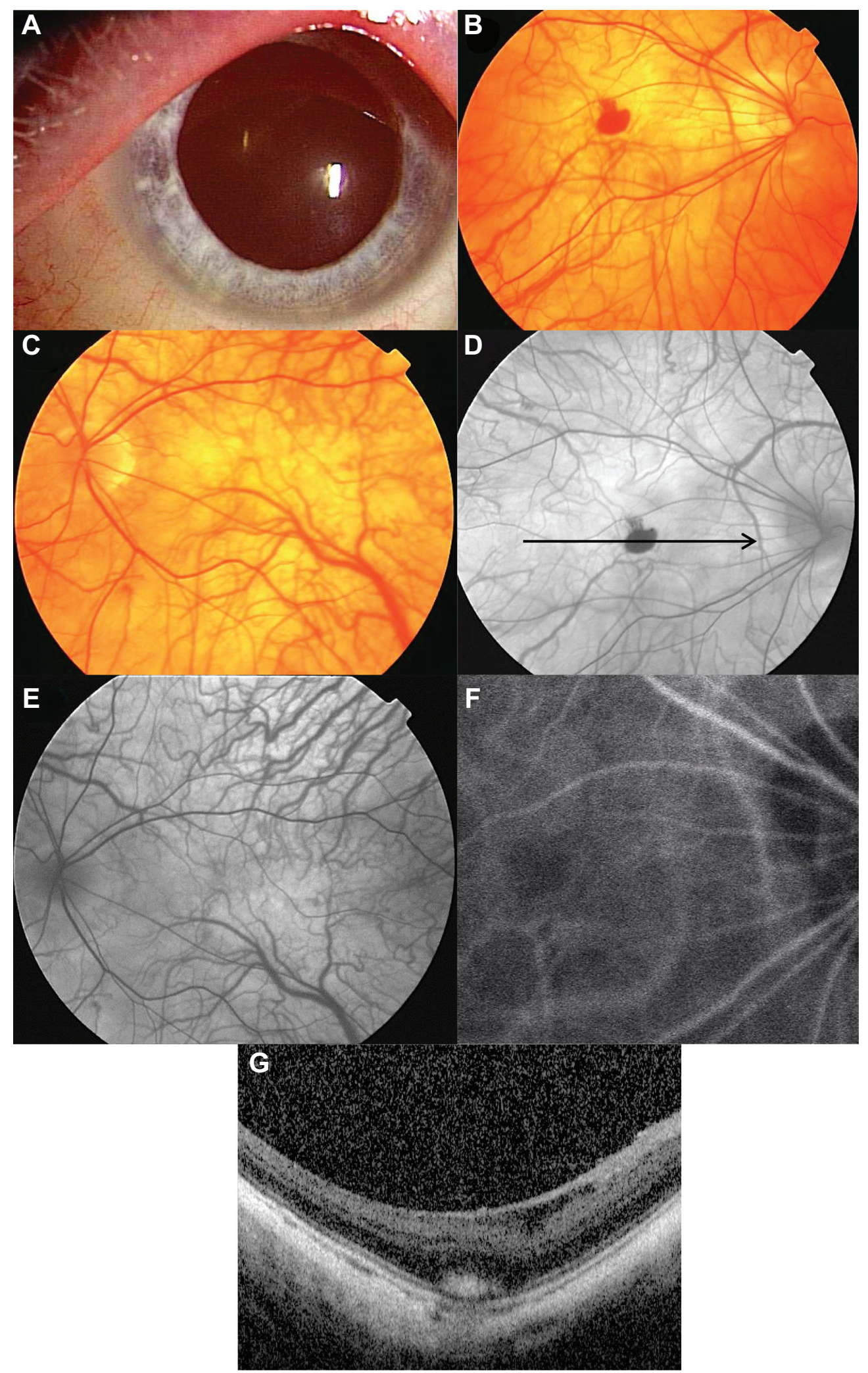

Figure I Findings in a 48-year-old Japanese man with oculocutaneous albinism. His best-corrected visual acuity was $2 / 100$ with -14.0 diopters in the right eye.

Notes: (A) External photograph of the right eye showing white cilia and a depigmented iris. (B, C) Fundus photographs at initial examination showing diffuse depigmentation. The right eye had a foveal hemorrhage (B). (D, E) Fluorescein angiographic images at the initial visit showing relative hypofluorescent retinal and choroidal vessels because of the lack of melanin pigment in the retinal pigment epithelium. The right eye has an area of hypofluorescence corresponding to the foveal hemorrhage (D). (F) Indocyanine green angiographic image of the right eye at the initial visit does not show any findings suggestive of choroidal neovascularization. (G) Optical coherence tomographic image corresponds to the arrow in Figure (D). A spectral-domain optical coherence tomographic image of the right eye at the initial visit shows an absence of the foveal pit, and the presence of a subretinal hyperreflective lesion corresponding to the foveal hemorrhage. 
diopters in the left eye. Ophthalmoscopy showed diffuse depigmentation in both eyes (Figure 1B and C). He was diagnosed with oculocutaneous albinism based on the systemic and ocular findings.

Ophthalmoscopy showed that his right eye had a foveal hemorrhage. Fluorescein angiography (FA) showed relatively hypofluorescent retinal and choroidal vessels because of the lack of a blocking effect by pigments in the retinal pigment epithelium (Figure 1D and E). FA also showed an area of hypofluorescence in the right fovea that corresponded with the foveal hemorrhage (Figure 1D). FA and indocyanine green angiography (IA, Figure $1 \mathrm{~F}$ ) did not show any evidence of choroidal neovascularization (CNV) in either eye. We could not obtain good quality optical coherence tomographic images because of the nystagmus in both eyes. However, the blurred optical coherence tomographic images showed an absence of the foveal pit in both eyes and the presence of a subretinal hyperreflective lesion corresponding to the foveal hemorrhage in the right eye (Figure 1G).

The foveal hemorrhage in the right eye spontaneously regressed and finally resolved at 3 months after onset (Figure 2). The best-corrected visual acuity of his right eye did not change after resolution of the hemorrhage, but he reported that his vision was better.

\section{Discussion}

A foveal hemorrhage in oculocutaneous albinism is relatively rare. Shinno et al reported a case of foveal hemorrhage in oculocutaneous albinism, ${ }^{4}$ and suggested that the foveal hemorrhage was due to $\mathrm{CNV}$ associated with high myopia. They came to this conclusion because FA and IA showed a hyperfluorescent lesion corresponding to CNV. However, in our case, FA and IA did not show CNV, although the patient had high myopia of -14.0 diopters accompanied by a staphyloma.

Oculocutaneous albinism is usually associated with foveal hypoplasia, ${ }^{5}$ and we were able to demonstrate foveal hypoplasia by the absence of a foveal pit in the optical coherence tomographic images. It was interesting to detect a foveal hemorrhage in an eye with foveal hypoplasia. The anatomical structure of the fovea, Bruch's membrane, and choriocapillaris in an eye with foveal hypoplasia may be different from that in normal eyes. However, a search of PubMed did not yield any previous publications mentioning the anatomical structure of the fovea in eyes with foveal hypoplasia.

Oculocutaneous albinism is often accompanied by high myopia. It is well known that a foveal hemorrhage in high myopic eyes without CNV is due to a rupture of Bruch's membrane and the choriocapillaris complex. The incidence of a foveal hemorrhage has been reported to be approximately $3 \%$ in highly myopic eyes. ${ }^{6}$ Our patient had a staphyloma with high myopia of -14.0 diopters, and this may have made the foveal tissues more fragile. Thus, we suggest that the foveal hemorrhage observed in our case was probably due to high myopia and it is likely that the foveal hypoplasia also contributed to the foveal hemorrhage.

In conclusion, a foveal hemorrhage is a rare condition in an eye with a foveal hypoplasia associated with albinism. The foveal hemorrhage in our case may be related to high myopia and also may be related to anatomical alterations associated with foveal hypoplasia.

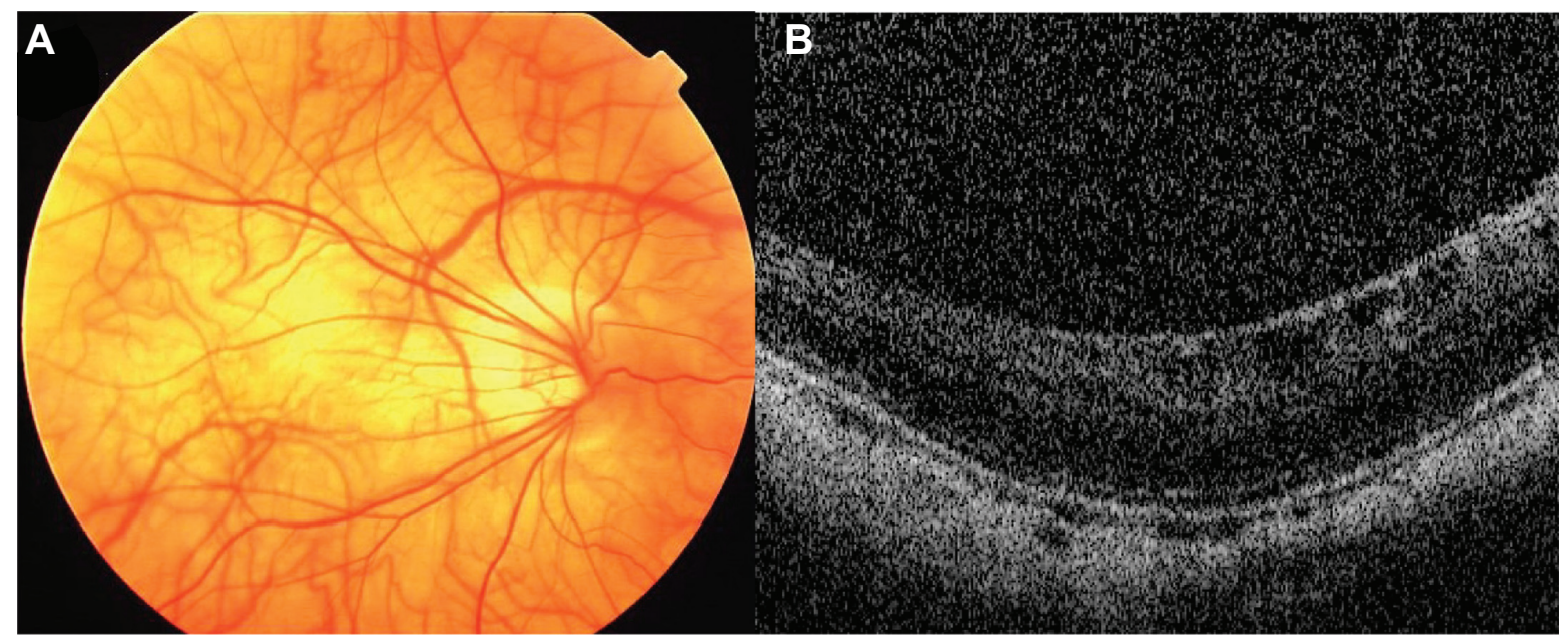

Figure 2 Three months after onset. The patient's best-corrected visual acuity has not improved.

Notes: (A) Fundus photograph of the right eye showing that the foveal hemorrhage has regressed. (B) Spectral-domain optical coherence tomographic image of the right eye showing an absence of a subretinal hyperreflective lesion corresponding to the foveal hemorrhage. 


\section{Disclosure}

The authors report no conflicts of interest in this work.

\section{References}

1. Mohammad S, Gottlob I, Kumar A, et al. The functional significance of foveal abnormalities in albinism measured using spectral-domain optical coherence tomography. Ophthalmology. 2011;118(8):1645-1652.

2. Chong GT, Farsiu S, Freedman SF, et al. Abnormal foveal morphology in ocular albinism imaged with spectral-domain optical coherence tomography. Arch Ophthalmol. 2009;127(1):37-44.

3. Rossi S, Testa F, Gargiulo A, et al. The role of optical coherence tomography in an atypical case of oculocutaneous albinism: a case report. Case Rep Ophthalmol. 2012;3(1):113-117.
4. Shinno M, Yumiko Y, Shoji K. [Choroidal neovascularization in a case of oculocutaneous albinism]. Rinsho Ganka. 2004;58(7):1245-1249. Japanese.

5. Thomas MG, Kumar A, Mohammad S, et al. Structural grading of foveal hypoplasia using spectral-domain optical coherence tomography a predictor of visual acuity? Ophthalmology. 2011;118(8):1653-1660.

6. Tokoro T. Explanatory factors of chorioretinal atrophy. In: Tokoro T, editor. Atlas of Posterior Fundus Changes in Pathologic Myopia. Tokyo, Japan: Springer Verlag; 1998.
Clinical Ophthalmology

\section{Publish your work in this journal}

Clinical Ophthalmology is an international, peer-reviewed journal covering all subspecialties within ophthalmology. Key topics include: Optometry; Visual science; Pharmacology and drug therapy in eye diseases; Basic Sciences; Primary and Secondary eye care; Patient Safety and Quality of Care Improvements. This journal is indexed on

Submit your manuscript here: http://www.dovepress.com/clinical-ophthalmology-journal

\section{Dovepress}

PubMed Central and CAS, and is the official journal of The Society of Clinical Ophthalmology (SCO). The manuscript management system is completely online and includes a very quick and fair peer-review system, which is all easy to use. Visit http://www.dovepress.com/ testimonials.php to read real quotes from published authors. 